\title{
Mathematical Reasoning of Treatment Principle Based on "Yin Yang Wu Xing" Theory in Traditional Chinese Medicine
}

\author{
Yingshan Zhang \\ School of Finance and Statistics, East China Normal University, Shanghai, China \\ E-mail: ysh_zhang@163.com \\ Received December 21, 2010; revised January 20, 2011; accepted January 28, 2011
}

\begin{abstract}
By using mathematical reasoning, this paper demonstrates the treatment principle: "Virtual disease is to fill his mother but real disease is to rush down his son" and "Strong inhibition of the same time, support the weak" based on "Yin Yang Wu Xing" Theory in Traditional Chinese Medicine (TCM). We defined two kinds of opposite relations and one kind of equivalence relation, introduced the concept of steady multilateral systems with two non-compatibility relations, and discussed its energy properties. Later based on the treatment of TCM and treated the healthy body as a steady multilateral system, it has been proved that the treatment principle is true. The kernel of this paper is the existence and reasoning of the non-compatibility relations in steady multilateral systems, and it accords with the oriental thinking model.
\end{abstract}

Keywords: Traditional Chinese Medicine, “Yin Yang Wu Xing” Theory, Steady Multilateral Systems, Opposite Relations, Side Effects, Medical and Drug Resistance Problem

\section{Main Differences between Traditional Chinese Medicine and Western Medicine}

Western medicine treats disease from Microscopic point of view, always destroys the original human being's balance, and has none beneficial to human's immunity. Western medicine can produce pollution to human's body, having strong side effects. Excessively using medicine can easily paralysis the human's immunity, which AIDS is a unique product of Western medicine. Using medicine too little can easily produce the medical and drug resistance problem.

Traditional Chinese Medicine (TCM) studies the world from the macroscopic point of view, and its target is in order to maintain the original balance of human being and in order to enhance the immunity. TCM believes that each medicine has one-third of drug. She never encourages patients to use medicine in long term. Traditional Chinese Medicine has over 5,000-year history. It almost has none side effects or medical and drug resistance problem [19].

After long period of practicing, Chinese ancient medical scientists use "Yin Yang Wu Xing" Theory exten- sively in the traditional treatment to explain the origin of life, human body, pathological changes, clinical diagnosis and prevention. It has become an important part of the Traditional Chinese Medicine. "Yin Yang Wu Xing" Theory has a strong influence to the formation and development of Chinese medicine theory. But, many Chinese and foreign scholars still have some questions on the reasoning of Traditional Chinese Medicine.

Zhang's theory, multilateral matrix theory [1] and multilateral system theory $[13,14,19]$, have given a new and strong mathematical reasoning method from macro (Global) analysis to micro (Local) analysis. He and his colleagues have made some mathematical models and methods of reasoning [2-17], which make the mathematical reasoning of TCM possible [13] based on "Yin Yang Wu Xing" Theory [18]. This paper will use steady multilateral systems to demonstrate the treatment principle of TCM: "Real disease is to rush down his son but virtual disease is to fill his mother" and "Strong inhibition of the same time, support the weak".

The article proceeds as follows. Section 2 contains basic concepts and main theorems of steady multilateral systems while the treatment principle of Traditional Chinese Medicine is demonstrated in Section 3 and 4. 
Conclusions are drawn in Section 5.

\section{Basic Concept of Steady Multilateral Systems}

In the real world, we are enlightened from some concepts and phenomena such as "biosphere", "food chain", "ecological balance" etc. With research and practice, by using the theory of multilateral matrices[1] and analyzing the conditions of symmetry [1-2] and orthogonality [3-5, $12,17]$ what a stable system must satisfy, in particular, with analyzing the basic conditions what a stable working procedure of good product quality must satisfy [11, 17], we are inspired and find some rules and methods, then present the logic model of analyzing stability of complex systems [7-10]—steady multilateral systems $[13,14,19]$. There are a number of essential reasoning methods based on the stable logic analysis model, such as "transition reasoning”, "atavism reasoning”, "genetic reasoning” etc.

\subsection{Equivalence Relations}

Let $V$ be a nonempty set and $x, y, z$ be their elements. We call it an equivalence relation, denoted $\sim$, if the following 3 conditions are all true:

1) Reflexive: $x \sim x$ for all $x$;

2) Symmetric: if $x \sim y$, then $y \sim x$;

3) Conveyable (Transitivity): if $x \sim y, y \sim z$, then $x \sim z$.

If there are some $\mathrm{x}, \mathrm{y}, \mathrm{z}$ such that at least one of the conditions above is true, the relation is called a compatibility relation. Any one of compatibility relations can be expanded into an equivalence relation [19].

Western Science only considers the reasoning under one Axiom system such that only researches on compatibility relation reasoning. However, there are many Axiom systems in Nature. Traditional Chinese Science (TCS, or Oriental Science) mainly researches the reasoning among many Axiom systems in Nature. Of course, she also considers the reasoning under one Axiom system but she only expands the reasoning as the equivalence relation reasoning [19].

\subsection{Two Kinds of Opposite Relations}

Equivalence relations, even compatibility relations, cannot portray the structure of the complex systems clearly. For example, assume that $A$ and $B$ are good friends and they have close relations. So are $B$ and $C$. However, you cannot get the conclusion that $A$ and $C$ are good friends. We denote $A \rightarrow B$ as that $A$ and $B$ have close relations. Then the example above can be denoted as: $A \rightarrow B, B \rightarrow$ $C$ do not imply $A \rightarrow C$, i.e., the relation $\rightarrow$ is a non- conveyable (or non-transitivity) relation, of course, a non-equivalence relation.

In the following, we consider two non-compatibility relations.

Let $V$ be a nonempty set and $x, y, z$ be none equal. There are two kinds of opposite relations, called neighboring relations $\rightarrow$ and alternate relations $\Rightarrow$, having the property:

1) If $x \rightarrow y, y \rightarrow z$, then $x \Rightarrow z$;

$\Leftrightarrow$ if $x \rightarrow y, x \Rightarrow z$, then $y \rightarrow z$;

$\Leftrightarrow$ if $x \Rightarrow z, y \rightarrow z$, then $x \rightarrow y$.

2) If $x \Rightarrow y, y \Rightarrow z$, then $z \rightarrow x$;

$\Leftrightarrow$ if $z \rightarrow x, x \Rightarrow y$, then $y \Rightarrow z$;

$\Leftrightarrow$ if $y \Rightarrow z, z \rightarrow x$, then $x \Rightarrow y$.

Two kinds of opposite relations cannot be exist separately.

Such reasoning can be expressed as follows:

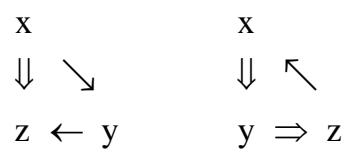

The first triangle reasoning is known as a jumpingtransition reasoning, while the second triangle reasoning is known as an atavism reasoning. Both neighboring relations and alternate relations are not compatibility relations, of course, none equivalence relations, called non-compatibility relations.

\subsection{Genetic Reasoning}

Let $V$ be a nonempty set and $x, y, z$ be not equal one another. If equivalence relations exist, neighboring relations, and alternate relations in $V$ at the same time, then a genetic reasoning is defined as follows:

1) if $x \sim y, y \rightarrow z$, then $x \rightarrow z$;

2) if $x \sim y, y \Rightarrow z$, then $x \Rightarrow z$;

3) if $x \rightarrow y, y \sim z$, then $x \rightarrow z$;

4) if $x \Rightarrow y, y \sim z$, then $x \Rightarrow z$.

\subsection{Multilateral Systems}

For a nonempty set $V$, if there exists at least an noncompatibility relation, then it is called a multilateral system about complexity $[13,14,19]$, or equivalently, a logic analysis model of complex systems [7-10].

Assume that there exist equivalence relations, neighboring relations, and alternate relations in system $V$, which satisfy genetic reasoning. If for every $x, y \in V$, at least there is one of the three relations between $x$ and $y$, and there are not $x \rightarrow y$ and $x \Rightarrow y$ at the same time, then $V$ is called a logic analysis model of complex systems, which is equivalent to the logic architecture of reasoning model of "Yin Yang" Theory in Ancient China. Obviously, $V$ is 
a multilateral system with two non-compatibility relations. In this paper, we only consider this multilateral system.

Theorem 2.1 For a multilateral system $V$ with two non-compatibility relations, $\forall x, y \in V$, only one of the following five relations is existent and correct:

$$
x \sim y, x \rightarrow y, x \leftarrow y, x \Rightarrow y, x \Leftarrow y .
$$

Theorem 2.2 For a multilateral system $V$ with two non-compatibility relations, $\forall x, y, z \in V$, the following reasoning holds:

1) if $x \rightarrow z, y \rightarrow z$, then $x \sim y$;

2) if $x \Rightarrow z, y \Rightarrow z$, then $x \sim y$;

3) if $x \rightarrow y, x \rightarrow z$, then $y \sim z$;

4) if $x \Rightarrow y, x \Rightarrow z$, then $y \sim z$.

\subsection{Steady Multilateral Systems}

The multilateral system $V$ is known as a steady multilateral system (or, a stable multilateral system) with two non-compatibility relations if there exists at least the chain $x_{1}, \cdots, x_{n} \in V$, which satisfy any one of the two conditions below:

$$
\begin{aligned}
& x_{1} \rightarrow x_{2} \rightarrow \cdots \rightarrow x_{n} \rightarrow x_{1} ; \\
& x_{1} \Rightarrow x_{2} \Rightarrow \cdots \Rightarrow x_{n} \Rightarrow x_{1} .
\end{aligned}
$$

Theorem 2.3 For a steady multilateral system $V$ with two non-compatibility relations, there exists five-length chain, and the length of the chain is integer times of 5.

Theorem 2.4 For a steady multilateral system $V$ with two non-compatibility relations, there exists a partition of $V$ as follows:

$$
\begin{gathered}
V=V_{1}+V_{2}+V_{3}+V_{4}+V_{5} ; \\
V_{i}=\left\{y \in V \mid y \sim x_{i}\right\}, \forall i=1, \cdots 5 .
\end{gathered}
$$

which $x_{1}, x_{2}, x_{3}, x_{4}, x_{5}$ is a chain. The notation that $V=V_{1}$ $+V_{2}+V_{3}+V_{4}+V_{5}$ means that $V=V_{1} \cup V_{2} \cup V_{3} \cup V_{4}$ $\cup V_{5}, V_{i} \cap V_{j}=\varnothing, \forall i \neq j$.

Theorem 2.5 The decomposition above for the steady multilateral system with two non-compatibility relations, there exist relations below Figure 1.

Theorem 2.6 For each element $x$ in a steady multilateral system $V$ with two non-compatibility relations, there exist five equivalence classes below:

$$
\begin{aligned}
& X=\{y \in V \mid y \sim x\}, \quad X_{S}=\{y \in V \mid x \rightarrow y\}, \\
& X_{K}=\{y \in V \mid x \Rightarrow y\}, \quad K_{X}=\{y \in V \mid y \Rightarrow x\}, \\
& S_{X}=\{y \in V \mid y \rightarrow x\},
\end{aligned}
$$

which the five equivalence classes have relations below Figure 2.

\section{Relationship Analysis of Steady Multilateral Systems}

\subsection{Energy of a Multilateral System}

Energy concept is an important concept in Physics. Now, we introduce this concept to the multilateral systems and use these concepts to deal with the multilateral system diseases.

In mathematics, a multilateral system is said to have energy (or dynamic) if there is a none negative function $\varphi(*)$ which makes every subsystem meaningful of the multilateral system.

For two subsystems $V_{i}$ and $V_{j}$ of multilateral system $V$, denote $V_{i} \rightarrow V_{j}$ (or $V_{i} \Rightarrow V_{j}$, or $V_{i} \sim V_{j}$ ) means $x_{i} \rightarrow x_{j}$, $x_{i} \in$ $V_{i}, x_{j} \in V_{j}$ (or $x_{i} \Rightarrow x_{j}, x_{i} \in V_{i}, x_{j} \in V_{j}$ or $x_{i} \sim x_{j}, x_{i} \in V_{i}$, $\left.x_{j} \in V_{j}\right)$.

For subsystems $V_{i}, V_{j}$ and $V_{i} \cup V_{j}$ where $V_{i} \cap V_{j}=\varnothing, i$ $\neq j$, let $\varphi\left(V_{i}\right)=\left|V_{i}\right|, \varphi\left(V_{j}\right)=\left|V_{j}\right|$ and $\varphi\left(V_{i} \cup V_{j}\right)=\left|V_{i} \cup V_{j}\right|$, where $\varphi\left(V_{i} \cup V_{j}\right)$ is the total energy of both $V_{i}$ and $V_{j}$.

For an equivalence relation $V_{i} \sim V_{j}$, if $\left|V_{i} \cup V_{j}\right|=\left|V_{i}\right|+$ $\left|V_{j}\right|$ (the normal state of the energy of $V_{i} \sim V_{j}$ ), then the equivalence relation $V_{i} \sim V_{j}$ is called that $V_{i}$ likes $V_{j}$ which means that $V_{i}$ is similar to $V_{j}$. In this case, the $V_{i}$ is also called the brother of $V_{j}$ while the $V_{j}$ is also called the brother of $V_{i}$. In the causal model, the $V_{i}$ is called the similar family member of $V_{j}$ while the $V_{j}$ is also called the similar family member of $V_{i}$. There are not any causal relation considered between $V_{i}$ and $V_{j}$.

For a neighboring relation $V_{i} \rightarrow V_{j}$, if $\left|V_{i} \cup V_{j}\right|>\left|V_{i}\right|+$ $\left|V_{j}\right|$ (the normal state of the energy of $V_{i} \rightarrow V_{j}$ ), then the neighboring relation $V_{i} \rightarrow V_{j}$ is called that $V_{i}$ bears (or loves) $V_{j}$ [or that $V_{j}$ is born (or loved) by $V_{i}$ ] which means that $V_{i}$ is beneficial on $V_{j}$ each other. In this case, the $V_{i}$ is called the mother of $V_{j}$ while the $V_{j}$ is called the son of $V_{i}$. In the causal model, the $V_{i}$ is called the beneficial cause of $V_{j}$ while the $V_{j}$ is called the beneficial effect of $V_{i}$.

For an alternate relation $V_{i} \Rightarrow V_{j}$, if $\left|V_{i} \cup V_{j}\right|<\left|V_{i}\right|+$ $\left|V_{j}\right|$ (the normal state of the energy of $V_{i} \Rightarrow V_{j}$ ), then the alternate relation $V_{i} \Rightarrow V_{j}$ is called as that $V_{i}$ kills $V_{j}$ (or that $V_{j}$ is killed by $V_{i}$ ) which means that $V_{i}$ is harmful on $V_{j}$ each other. In this case, the $V_{i}$ is called the bane of $V_{j}$ while the $V_{j}$ is called the prisoner of $V_{i}$. In the causal model, the $V_{i}$ is called the harmful cause of $V_{j}$ while the $V_{j}$ is called the harmful effect of $V_{i}$.

In the future, unless stated otherwise, any equivalence relation is the liking relation, any neighboring relation is the bearing relation (or the loving relation), and any alternate relation is the killing relation.

Suppose $V$ is a steady multilateral system having energy, then during normal operation, its energy function for any subsystem of the multilateral system has an average (or expected value in Statistics), the state is called 
normal when the energy function is nearly to the average. Normal state is the better state.

A subsystem of a multilateral system is called not running properly (or disease, abnormal), if the energy deviation from the average of the subsystems is too large, the high [real disease] or the low [virtual disease].

In a subsystem of a multilateral system being not running properly, if the energy of this sub-system is increased or decreased by using external forces and returned to its average (or its expected value), this method is called intervention (or making a medical treatment) to the multilateral system.

The purpose of intervention is to make the multilateral system return to normal state. The method of intervention is to increase or to decrease the energy of a subsystem.

What kind of treatment should follow the principle to treat it? Western medicine emphasizes direct treatment, but the indirect treatment of oriental medicine (or Traditional Chinese Medicine) is required. In mathematics, which is more reasonable?

Based on this idea, many issues are worth further discussion. For example, if an intervention treatment has been done to a multilateral system, what situation will happen?

\subsection{Intervention Rule of a Multilateral System}

For a steady multilateral system $V$ with two non-compatibility relations, suppose that there is an external force (or an intervening force) on the subsystem $X$ of $V$ which makes the energy $\varphi(X)$ of $X$ changed by the increment $\Delta \varphi(X)$, then the energies $\varphi\left(X_{S}\right), \varphi\left(X_{K}\right), \varphi\left(K_{X}\right)$, $\varphi\left(S_{X}\right)$ of other subsystems $X_{S}, X_{K}, K_{X}, S_{X}$ (defined in Theorem 2.6) of $\mathrm{V}$ will be changed by the increments $\Delta \varphi\left(X_{S}\right), \Delta \varphi\left(X_{K}\right), \Delta \varphi\left(K_{X}\right)$ and $\Delta \varphi\left(S_{X}\right)$, respectively.

It is said that a multilateral system has the capability of intervention reaction if the multilateral system has capability to response the intervention force.

If a subsystem $X$ of multilateral system $V$ is intervened, then the energies $\Delta \varphi\left(X_{S}\right)$ and $\Delta \varphi\left(S_{X}\right)$ of the subsystems $X_{S}$ and $S_{X}$ which have neighboring relations to $X$ will change in the same direction of the force outside on $X$.We call them beneficiaries. But the energies $\Delta \varphi\left(X_{K}\right)$ and $\Delta \varphi\left(K_{X}\right)$ of the subsystems $X_{K}$ and $K_{X}$ which have alternate relations to $X$ will change in the opposite direction of the force outside on $X$. We call them victims.

Furthermore, in general, there is an essential principle of intervention: any one of energies $\Delta \varphi\left(X_{S}\right)$ and $\Delta \varphi\left(S_{X}\right)$ of beneficial subsystems $X_{S}$ and $S_{X}$ of $X$ changes in the same direction of the force outside on $X$, and any one of energies $\Delta \varphi\left(X_{K}\right)$ and $\Delta \varphi\left(K_{X}\right)$ of harmful subsystems $X_{K}$ and $K_{X}$ of $X$ changes in the opposite direction of the force outside on $X$. The changed size of the energy $\Delta \varphi\left(X_{S}\right)$ (or $\Delta \varphi\left(S_{X}\right)$ ) is equal to that of $\Delta \varphi\left(X_{K}\right)$ (or $\Delta \varphi\left(K_{X}\right)$ ), but the direction opposite.

Intervention Rule: In the case of virtual disease, the treatment method of intervention is to increase the energy. If the treatment has been done on $X$, the energy increment (or, increase degree) $\left|\Delta \varphi\left(X_{S}\right)\right|$ of the son $X_{S}$ of $X$ is greater than the energy increment (or, increase degree) $\left|\Delta \varphi\left(S_{X}\right)\right|$ of the mother $S_{X}$ of $X$, i.e., the best benefit is the son $X_{S}$ of $X$. But the energy decrease degree $\left|\Delta \varphi\left(X_{K}\right)\right|$ of the prisoner $X_{K}$ of $X$ is greater than the energy decrease degree $\left|\Delta \varphi\left(K_{X}\right)\right|$ of the bane $K_{X}$ of $X$, i.e., the worst victim is the prisoner $X_{K}$ of $X$.

In the case of real disease, the treatment method of intervention is to decrease the energy. If the treatment has been done on $X$, the energy decrease degree $\left|\Delta \varphi\left(S_{X}\right)\right|$ of the mother $S_{X}$ of $X$ is greater than the energy decrease degree $\left|\Delta \varphi\left(X_{S}\right)\right|$ of the son $X_{S}$ of $X$, i.e., the best benefit is the mother $S_{X}$ of $X$. But the energy increment (or, increase degree) $\left|\Delta \varphi\left(K_{X}\right)\right|$ of the bane $K_{X}$ of $X$ is greater than the energy increment (or, increase degree) $\left|\Delta \varphi\left(X_{K}\right)\right|$ of the prisoner $X_{K}$ of $X$, i.e., the worst victim is the bane $K_{X}$ of $X$.

In mathematics, the changing laws are as follows.

1) If $\Delta \varphi(X)=\Delta>0$, then $\Delta \varphi\left(X_{S}\right)=\rho_{1} \Delta, \Delta \varphi\left(X_{K}\right)=-\rho_{1} \Delta$, $\Delta \varphi\left(K_{X}\right)=-\rho_{2} \Delta, \Delta \varphi\left(S_{X}\right)=\rho_{2} \Delta$;

2) If $\Delta \varphi(X)=-\Delta<0$, then $\Delta \varphi\left(X_{S}\right)=-\rho_{2} \Delta, \Delta \varphi\left(X_{K}\right)=$ $\rho_{2} \Delta, \Delta \varphi\left(K_{X}\right)=\rho_{1} \Delta, \Delta \varphi\left(S_{X}\right)=-\rho_{1} \Delta$;

where $1 \geq \rho_{1} \geq \rho_{2} \geq 0$. Both $\rho_{1}$ and $\rho_{2}$ are called intervention reaction coefficients, which are used to represent the capability of intervention reaction. The larger $\rho_{1}$, the better the capability of intervention reaction. The state $\rho_{1}=1$ is the best state but the state $\rho_{1}=0$ is the worst state.

Medical and drug resistance problem is that such a question, beginning more appropriate medical treatment, but is no longer valid after a period. It is because the capability of intervention reaction is bad, i.e., the intervention reaction coefficients $\rho_{1}$ and $\rho_{2}$ are too small. In the state $\rho_{1}=1$, any medical and drug resistance problem is non-existence but in the state $\rho_{1}=0$, medical and drug resistance problem is always existence. At this point, the paper advocates the principle of treatment to avoid medical and drug resistance problems.

This intervention rule is similar to force and reaction in Physics.

\subsection{Self-Protection Rule of a Multilateral System}

If there is an intervening force on the subsystem $X$ of a steady multilateral system $V$ which makes the energy $\varphi(X)$ changed by increment $\Delta \varphi(X)$ such that the energies $\varphi\left(X_{S}\right)$, $\varphi\left(X_{K}\right), \varphi\left(K_{X}\right), \varphi\left(S_{X}\right)$ of other subsystems $X_{S}, X_{K}, K_{X}, S_{X}$ (defined in Theorem 2.6) of $V$ will be changed by the 
increments $\Delta \varphi\left(X_{S}\right), \Delta \varphi\left(X_{K}\right), \Delta \varphi\left(K_{X}\right), \Delta \varphi\left(S_{X}\right)$, respectively, then can the multilateral system $V$ have capability to protect the worst victim to restore?

It is said that the steady multilateral system has the capability of self-protection if the multilateral system has capability to protect the worst victim to restore. The capability of self-protection of the steady multilateral system is said to be better if the multilateral system has capability to protect the all victims to restore.

In general, there is an essential principle of self-protection: any harmful subsystem of $X$ should be protected by using the same intervention force but any beneficial subsystem of $X$ should not.

Self-protection Rule: in the case of virtual disease, the treatment method of intervention is to increase the energy. If the treatment has been done on $X$, the worst victim is the prisoner $X_{K}$ of $X$. Thus, the treatment of self-protection is to restore the prisoner $X_{K}$ of $X$ and the restoring method of self-protection is to increase the energy $\varphi\left(X_{K}\right)$ of the prisoner $X_{K}$ of $X$ by using the intervention force on $X$ according to the intervention rule.

In the case of real disease, the treatment method of intervention is to decrease the energy. If the treatment has been done on $X$, the worst victim is the bane $K_{X}$ of $X$. Thus, the treatment of self-protection is to restore the bane $K_{X}$ of $X$ and the restoring method of self-protection is to decrease the energy $\varphi\left(K_{X}\right)$ of the bane $K_{X}$ of $X$ by using the same intervention force on $X$ according to the intervention rule.

In mathematics, the following self-protection laws hold.

1) If $\Delta \varphi(X)=\Delta>0$, then the energy of subsystem $X_{K}$ will decrease the increment $\left(-\rho_{1} \Delta\right)$, which is the worst victim. So the capability of self-protection increases the energy of subsystem $X_{K}$ by increment $\left(\rho_{1} \Delta\right)$ in order to restore the worst victim $X_{K}$ by using the same intervention force on $X$ according to the intervention rule.

2) If $\Delta \varphi(X)=-\Delta<0$, then the energy $\Delta \varphi\left(K_{X}\right)$ of subsystem $K_{X}$ will increase the increment $\left(\Delta \varphi\left(K_{X}\right)=\rho_{1} \Delta\right)$, which is the worst victim. So the capability of self-protection decreases the energy of subsystem $K_{X}$, by the same size to $\Delta \varphi\left(K_{X}\right)$ but the direction opposite, i.e., by increment $\left.\Delta \varphi\left(X_{K}\right)_{1}=-\rho_{1} \Delta\right)$, in order to restore the worst victim $K_{X}$ by using the same intervention force on $X$ according to the intervention rule.

The self-protection rule can be explained as: the general principle of self-protection subsystem is the most affected is protected firstly; the protection method is in the same way to the intervention force.

Theorem 3.1 Suppose that a steady multilateral system $V$ which has energy and capability of self-protection is with intervention reaction coefficients $\rho_{1}$ and $\rho_{2}$. If the capability of self-protection can make the subsystem $X_{K}$ to be restored, then the following statements are true.

1) In the case of virtual disease, the treatment method is to increase the energy. If an intervention force on the subsystem $X$ of steady multilateral system $V$ is implemented such that its energy $\varphi(X)$ has been changed by increment $\Delta \varphi(X)=\Delta>0$, then all five subsystems will be changed finally by the increments as follows:

$$
\begin{aligned}
& \Delta \varphi(X)_{2}=\Delta \varphi(X)+\Delta \varphi(X)_{1}=\left(1-\rho_{2} \rho_{1}\right) \Delta>0, \\
& \Delta \varphi\left(X_{S}\right)_{2}=\Delta \varphi\left(X_{S}\right)+\Delta \varphi\left(X_{S}\right)_{1}=\left(\rho_{1}+\rho_{2} \rho_{1}\right) \Delta>0, \\
& \Delta \varphi\left(X_{K}\right)_{2}=\Delta \varphi\left(X_{K}\right)+\Delta \varphi\left(X_{K}\right)_{1}=\left(-\rho_{1}+\rho_{1}\right) \Delta=0, \\
& \Delta \varphi\left(K_{X}\right)_{2}=\Delta \varphi\left(K_{X}\right)+\Delta \varphi\left(K_{X}\right)_{1}=-\left(\rho_{2}-\rho_{1}^{2}\right) \Delta, \\
& \Delta \varphi\left(S_{X}\right)_{2}=\Delta \varphi\left(S_{X}\right)+\Delta \varphi\left(S_{X}\right)_{1}=\left(\rho_{2}-\rho_{1}^{2}\right) \Delta, \\
& \forall \Delta \varphi(X)=\Delta>0 .
\end{aligned}
$$

2) In the case of real disease, the treatment method is to decrease the energy. If an intervention force on the subsystem $X$ of steady multilateral system $V$ is implemented such that its energy $\varphi(X)^{\prime}$ has been changed by increment $\Delta \varphi(X)^{\prime}=-\Delta<0$, then all five subsystems will be changed finally by the increments as follows:

$$
\begin{aligned}
& \Delta \varphi(X)_{2}^{\prime}=\Delta \varphi(X)^{\prime}+\Delta \varphi(X)_{1}^{\prime}=-\left(1-\rho_{2} \rho_{1}\right) \Delta<0, \\
& \Delta \varphi\left(X_{S}\right)_{2}^{\prime}=\Delta \varphi\left(X_{S}\right)^{\prime}+\Delta \varphi\left(X_{S}\right)_{1}^{\prime}=-\left(\rho_{2}-\rho_{1}^{2}\right) \Delta, \\
& \Delta \varphi\left(X_{K}\right)_{2}^{\prime}=\Delta \varphi\left(X_{K}\right)^{\prime}+\Delta \varphi\left(X_{K}\right)_{1}^{\prime}=\left(\rho_{2}-\rho_{1}^{2}\right) \Delta, \\
& \Delta \varphi\left(K_{X}\right)_{2}^{\prime}=\Delta \varphi\left(K_{X}\right)^{\prime}+\Delta \varphi\left(K_{X}\right)_{1}^{\prime}=\left(\rho_{1}-\rho_{1}\right) \Delta=0, \\
& \Delta \varphi\left(S_{X}\right)_{2}^{\prime}=\Delta \varphi\left(S_{X}\right)^{\prime}+\Delta \varphi\left(S_{X}\right)_{1}^{\prime}=-\left(\rho_{1}+\rho_{2} \rho_{1}\right) \Delta<0, \\
& \forall \Delta \varphi(X)^{\prime}=-\Delta<0,
\end{aligned}
$$

where the $\Delta \varphi(*)_{1}$ 's and $\Delta \varphi(*)_{1}{ }^{\prime}$ 's are the increments under the capability of self-protection.

Corollary 3.1 Suppose that a steady multilateral system $V$ which has energy and capability of self-protection is with intervention reaction coefficients $\rho_{1}$ and $\rho_{2}$. Then the capability of self-protection can make both subsystems $X_{K}$ and $K_{X}$ to be restored at the same time, i.e., the capability of self-protection is better, if and only if $\rho_{2}=$ $\rho_{1}^{2}$.

Side effects of medical problems were the question: in the medical process, destroyed the normal balance of a normal system which is not falling-ill system or intervening system. By Theorem 3.1 and Corollary 3.1, it can be seen that a necessary and sufficient condition is $\rho_{2}=$ $\rho_{1}^{2}$ if the capability of self-protection of the steady multilateral system is better, i.e., the multilateral system has capability to protect all victims to restore. At this point, the paper advocates the principle to avoid any side effects of treatment. 


\subsection{Mathematical Reasoning of Treatment Principle by Using the Neighboring Relations of Steady Multilateral Systems}

Treatment principle by using the neighboring relations of steady multilateral systems is "Virtual disease is to fill his mother but real disease is to rush down his son". In order to show the rationality of the treatment principle, it is needed to prove the following theorems.

Theorem 3.2 Suppose that a steady multilateral system $V$ which has energy and capability of self-protection is with intervention reaction coefficients $\rho_{1}$ and $\rho_{2}$ satisfying $\rho_{2}=\rho_{1}^{2}$. Then the following statements are true.

In the case of virtual disease, if an intervention force on the subsystem $X$ of steady multilateral system $V$ is implemented such that its energy $\varphi(X)$ increases the increment $\Delta \varphi(X)=\Delta>0$, then the subsystems $S_{X}, X_{K}$ and $K_{X}$ can be restored at the same time, but the subsystems $X$ and $X_{S}$ will increase their energies finally by the increments

$$
\begin{aligned}
\Delta \varphi(X)_{2} & =\left(1-\rho_{1} \rho_{2}\right) \Delta \varphi(X)=\left(1-\rho_{1}^{3}\right) \Delta \varphi(X) \\
& =\left(1-\rho_{1}^{3}\right) \Delta
\end{aligned}
$$

and

$$
\begin{aligned}
\Delta \varphi\left(X_{S}\right)_{2} & =\left(\rho_{1}+\rho_{1} \rho_{2}\right) \Delta \varphi(X)=\left(\rho_{1}+\rho_{1}^{3}\right) \Delta \varphi(X) \\
& =\left(\rho_{1}+\rho_{1}^{3}\right) \Delta,
\end{aligned}
$$

respectively.

On the other hand, in the case of real disease, if an intervention force on the subsystem $X$ of steady multilateral system $V$ is implemented such that its energy $\varphi(X)^{\prime}$ decreases, i.e., by the increment $\Delta \varphi(X)^{\prime}=-\Delta<0$, the subsystems $X_{S}, X_{K}$ and $K_{X}$ can also be restored at the same time, and the subsystems $X$ and $S_{X}$ will decrease their energies finally, i.e., by the increments

$$
\begin{aligned}
\Delta \varphi(X)_{2}^{\prime} & =\left(1-\rho_{1} \rho_{2}\right) \Delta \varphi(X)^{\prime}=\left(1-\rho_{1}^{3}\right) \Delta \varphi(X)^{\prime} \\
& =-\left(1-\rho_{1}^{3}\right) \Delta
\end{aligned}
$$

and

$$
\begin{aligned}
\Delta \varphi\left(S_{X}\right)_{2}^{\prime} & =\left(\rho_{1}+\rho_{1} \rho_{2}\right) \Delta \varphi(X)^{\prime}=\left(\rho_{1}+\rho_{1}^{3}\right) \Delta \varphi(X)^{\prime} \\
& =-\left(\rho_{1}+\rho_{1}^{3}\right) \Delta,
\end{aligned}
$$

respectively.

Theorem 3.3 For a steady multilateral system $V$ which has energy and capability of self-protection, assume intervention reaction coefficients are $\rho_{1}$ and $\rho_{2}$ which satisfy $\rho_{2}=\rho_{1}^{2}$ and $\rho_{1} \geq 0.5897545123$. Then the following statements are true.

1) If an intervention force on the subsystem $X$ of steady multilateral system $V$ is implemented such that its energy $\varphi(X)$ has been changed by increment $\Delta \varphi(X)=\Delta>$ 0 , then the final increment $\left(\rho_{1}+\rho_{1}^{3}\right) \Delta$ of the energy $\varphi\left(X_{S}\right)$ of the subsystem $X_{S}$ changed is greater than the final increment $\left(1-\rho_{1}^{3}\right) \Delta$ of the energy $\varphi(X)$ of the subsystem $X$ changed based on the capability of self-protection.

2) If an intervention force on the subsystem $X$ of steady multilateral system $V$ is implemented such that its energy $\varphi(X)$ has been changed by increment $\Delta \varphi(X)=-\Delta<0$, then the final increment $-\left(\rho_{1}+\rho_{1}^{3}\right) \Delta$ of the energy $\varphi\left(S_{X}\right)$ of the subsystem $S_{X}$ changed is less than the final increment $-\left(1-\rho_{1}^{3}\right) \Delta$ of the energy $\varphi(X)$ of the subsystem $X$ changed based on the capability of self-protection.

Corollary 3.2 For a steady multilateral system $V$ which has energy and capability of self-protection, intervention reaction coefficients are $\rho_{1}$ and $\rho_{2}$ which satisfy $\rho_{2}=\rho_{1}^{2}$ and $\rho_{1}<0.5897545123$. Then the following statements are true.

1) In the case of virtual disease, if an intervention force on the subsystem $X$ of steady multilateral system $V$ is implemented such that its energy $\varphi(X)$ has been changed by increment $\Delta \varphi(X)=\Delta>0$, then the final increment $\left(\rho_{1}+\rho_{1}^{3}\right) \Delta$ of the energy $\varphi\left(X_{S}\right)$ of the subsystem $X_{S}$ changed is less than the final increment $\left(1-\rho_{1}^{3}\right) \Delta$ of the energy $\varphi(X)$ of the subsystem $X$ changed based on the capability of self-protection.

2) In the case of real disease, if an intervention force on the subsystem $X$ of steady multilateral system $V$ is implemented such that its energy $\varphi(X)$ has been changed by increment $\Delta \varphi(X)=-\Delta<0$, then the final increment $-\left(\rho_{1}+\rho_{1}^{3}\right) \Delta$ of the energy $\varphi\left(S_{X}\right)$ of the subsystem $S_{X}$ changed is greater than the final increment $-\left(1-\rho_{1}^{3}\right) \Delta$ of the energy $\varphi(X)$ of the subsystem $X$ changed based on the capability of self-protection.

\subsection{Mathematical Reasoning of Treatment Principle by Using the Alternate Relations of Steady Multilateral Systems}

Treatment principle by using the alternate relations of steady multilateral systems is "Strong inhibition of the same time, support the weak". In order to show the rationality of the treatment principle, it needed to prove the following theorem.

Theorem 3.4 Suppose that a steady multilateral system $V$ which has energy and capability of self-protection is with intervention reaction coefficients $\rho_{1}$ and $\rho_{2}$ satisfying $\rho_{2}=\rho_{1}^{2}$. Assume there are two subsystems $X$ and $X_{K}$ of $V$ with an alternate relation such that $X$ encounters virtual disease, and at the same time, $X_{K}$ befalls real disease. Then the following statements are true.

If an intervention force on the subsystem $X$ of steady multilateral system $V$ is implemented such that its energy $\varphi(X)$ has been changed by increment $\Delta \varphi(X)=\Delta>0$, and 
at the same time, another intervention force on the subsystem $X_{K}$ of steady multilateral system $V$ is also implemented such that its energy $\varphi\left(X_{K}\right)$ has been changed by increment $\Delta \varphi\left(X_{K}\right)=-\Delta<0$, then all other subsystems: $S_{X}$, $K_{X}$ and $X_{S}$ can be restored at the same time, and the subsystems $X$ and $X_{K}$ will decrease and increase their energies by the same size but the direction opposite, i.e., by the final increments

$$
\begin{aligned}
\Delta \varphi(X)_{3} & =\left(1-\rho_{1} \rho_{2}\right) \Delta \varphi(X)=\left(1-\rho_{1}^{3}\right) \Delta \varphi(X) \\
& =\left(1-\rho_{1}^{3}\right) \Delta
\end{aligned}
$$

and

$$
\begin{aligned}
\Delta \varphi\left(X_{K}\right)_{3} & =\left(1-\rho_{1} \rho_{2}\right) \Delta \varphi\left(X_{K}\right)=\left(1-\rho_{1}^{3}\right) \Delta \varphi\left(X_{K}\right) \\
& =-\left(1-\rho_{1}^{3}\right) \Delta
\end{aligned}
$$

respectively.

These theorems can been found in [7-10] and [13,14]. Figures 1 and 2 in Theorems 2.5 and 2.6 are the Figures of "Wu Xing" Theory in Ancient China. The steady multilateral system $V$ with two non-compatibility relations is equivalent to the logic architecture of reasoning model of "Yin Yang Wu Xing" Theory in Ancient China.

\section{Rationality of Treatment Principle of Traditional Chinese Medicine and "Yin Yang Wu Xing” Theory}

\subsection{Traditional Chinese Medicine and "Yin Yang Wu Xing” Theory}

Ancient Chinese "Yin Yang Wu Xing" Theory has been surviving for several thousands of years without dying out, proving it reasonable to some extent. If we regard as the same category, the neighboring relation as beneficial, harmony, obedient, loving, etc., and the alternate relation $\Rightarrow$ as harmful, conflict, ruinous, killing, etc., then the above defined stable logic analysis model is similar to the logic architecture of reasoning of "Yin Yang Wu Xing”. Both "Yin" and "Yang” mean that there are two opposite relations in the world: harmony or loving $\rightarrow$ and conflict or killing $\Rightarrow$, as well as a general equivalent category $\sim$. There is only one of three relations $\sim, \rightarrow$ and $\Rightarrow$ between every two objects. Everything $(X \neq \varnothing)$ makes something $\left(X_{S} \neq \varnothing\right)$, and is made by something $\left(S_{X} \neq \varnothing\right)$; Everything restrains something $\left(X_{K} \neq\right.$ $\varnothing)$, and is restrained by something $\left(K_{X} \neq \varnothing\right)$; i.e., one thing overcomes another thing and one thing is overcome by another thing. The ever changing world $V$, following the relations: $\sim, \rightarrow$ and $\Rightarrow$, must be divided into five categories by the equivalent relation $\sim$, being called " $W u$ $X i n g$ ": wood $(X)$, fire $\left(X_{S}\right)$, earth $\left(X_{K}\right)$, gold $\left(K_{X}\right)$ and wa- ter $\left(S_{X}\right)$.

The relationship among the "Wu Xing" is to be "neighbor is friend":

$$
\operatorname{wood}(X) \rightarrow \operatorname{fire}\left(X_{S}\right) \rightarrow \operatorname{earth}\left(X_{K}\right) \rightarrow \operatorname{gold}\left(K_{X}\right) \rightarrow \text { wa- }
$$

$\operatorname{ter}\left(S_{X}\right) \rightarrow \operatorname{wood}(X)$,

and to be "alternate is foe":

$\operatorname{wood}(X) \Rightarrow \operatorname{earth}\left(X_{K}\right) \Rightarrow \operatorname{water}\left(S_{X}\right) \Rightarrow \operatorname{fire}\left(X_{S}\right) \Rightarrow$ $\operatorname{gold}\left(K_{X}\right) \Rightarrow \operatorname{wood}(X)$.

On the other words, $V=X+X_{S}+X_{K}+K_{X}+S_{X}$ satisfying

and

$$
X \rightarrow X_{S} \rightarrow X_{K} \rightarrow K_{X} \rightarrow S_{X} \rightarrow X
$$

$$
X \Rightarrow X_{K} \Rightarrow S_{X} \Rightarrow X_{S} \Rightarrow K_{X} \Rightarrow X
$$

where elements in the same category are equivalent to one another. We can see, from this, the ancient Chinese theory "Yin Yang Wu Xing" is a reasonable logic analysis model to identify the stability and relationship of complex systems, i.e., it is a steady multilateral system.

Traditional Chinese Medicine (TCM) firstly uses the verifying relationship method of "Yin Yang Wu Xing" Theory to explain the relationship between organ of human body and environment. Secondly, based on "Yin Yang Wu Xing" Theory, the relations of physiological processes of human body can be shown by the neighboring relation and alternate relation of five subsets. Thus a normal human's body can be shown as a steady multilateral system. Loving relation in TCM can be explained as the neighboring relation, called "Sheng”. Killing relation in TCM can be explained as the alternate relation, called

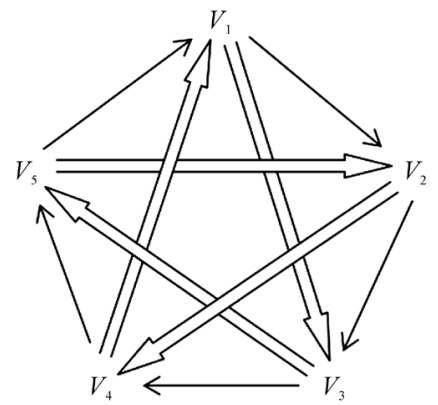

Figure 1. Uniquely steady architecture: “Wu Xing”.

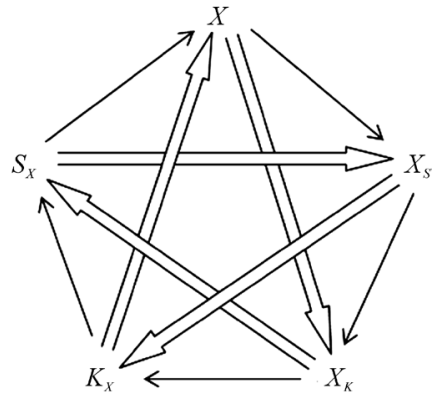

Figure. 2 The method of finding “Wu Xing”. 
"Ke". Constraints and conversion between five subsets are equivalent to the two kinds of triangle reasoning. So a normal human's body can be classified into five equivalence classes. It has been shown in Theorems 2.1-2.6 that the classification of five subsets is quite possible based on the mathematical logic. To make sure the characteristics of the five subsets is reasonable or not, it needs more research work. It has been also shown in Theorems 3.2-3.4 that the logical basis of TCM is a steady multilateral system.

The gas ["Chi”, or "Qi", energy of life] of TCM means the energy in a steady multilateral system.

There are two kinds of diseases in TCM: real disease and virtual disease. They generally mean the subsystem is abnormal (or disease), its energy ["Chi”, or "Qi", energy of life] is too high or too low.

The treatment method of TCM is to "Xie Qi" which means to rush down the energy if a real disease is treated, or to "Bu Qi" which means to fill the energy if a virtual disease is treated. Like intervening the subsystem, decrease when the energy is too high, increase when the energy is too low.

Both the capability of intervention reaction and the capability of self-protection of the multilateral system are equivalent to the Immunization of TCM. This capability is really exist. Its target is to protect other organs while treating one organ.

\subsection{Treatment Principle if Only One Organ of the Human Body System Falls Ill}

If we always intervene the abnormal organ of the human body system directly, the intervention method always destroy the balance of the human body systems because it is having strong side effects to the mother or the son of the organ which is non-disease system by using Theorem 3.2. The intervening directly method also decreases the capability of intervention reaction $\rho_{1}$, because the method which doesn't use the capability of intervention reaction makes the $\rho_{1}$ near to 0 . The state $\rho_{1}=0$ is the worst state of the human body system, namely AIDS. On the way, the resistance problem will occur since any medicine or treatment has little effect for small $\rho_{1}$.

However, by Corollary 3.2, it will even be better if we intervene subsystem $X$ itself directly while $\rho_{1}<$ 0.5897545123 , i.e. $\rho_{1}+\rho_{1}{ }^{2}<0.9375648971$. It can be explained that if a multilateral system which has a poor capability of intervention reaction, then it is better to intervene the subsystem $X$ itself directly than indirectly. However, similar to above, the intervening directly method always destroys the balance of multilateral systems such that there is at least one side effect occurred. Moreover, the intervening directly method is also harm- ful to the capability of intervention reaction and might causes the medical and drug resistance problem. Therefore, the intervention method directly can be used in case $\rho_{1}<0.5897545123$ but should be used as little as possible.

If we always intervene in the abnormal organ of the human body system indirectly, the intervention method can be to maintain the balance of the human body system because it has not any side effects to all other organs which are not both the disease organ and the intervened organ by using Theorem 3.2. The intervening indirectly method also increase the capability of intervention reaction because the method of using the intervention reaction makes the $\rho_{1}$ near to 1 . The state $\rho_{1}=1$ is the best state of the human body system. On the way, it almost has none medical and drug resistance problem since any medicine is possible good for some large $\rho_{1}$.

Overall, the human body system satisfies the intervention rule and the self-protection rule. It is said healthy while intervention reaction coefficient $\rho_{1}$ satisfies $\rho_{1}>$ 0.5897545123. In logic and practice, it's reasonable $\rho_{1}+$ $\rho_{2}$ near to 1 . In case: $\rho_{1}+\rho_{2}=1$, all the energy for intervening human body organ can transmit to other human body organs which have neighboring relations or alternate relations with the intervening human body organ. The healthy condition $\rho_{1}>0.5897545123$ can be satisfied when $\rho_{2}=\rho_{1}{ }^{2}$ for a healthy human body since $\rho_{1}+\rho_{2}$ $=1$ implies $\rho_{1}=(\sqrt{5}-1) / 2 \approx 0.618>0.5897545123$. If this assumptions is set up, then the treatment principle: "Real disease is to rush down his son and virtual disease is to fill his mother" based on "Yin Yang Wu Xing" Theory of TCM, is quite reasonable.

On the other hand, in TCM, real disease and virtual disease have their reasons. The bear organ $X_{S}$ causes real disease of $X$, while the born organ $S_{X}$ causes virtual disease of $X$. Although the reason cannot be proved easily in mathematics or experiments, the treatment method under the assumption is quite equal to the treatment method in the intervention indirectly. It has also proved that the treatment principle is true from the other side.

\subsection{Treatment Principle if Two Organs with the Loving Relation of the Human Body System Encounter Sick}

Suppose that the two organs $X$ and $X_{S}$ of the human body system are abnormal (or disease). In the human body of relations between non-compatible with the constraints, only two situations may occur:

1) $X$ encounters virtual disease, and at the same time, $X_{S}$ befalls virtual disease, i.e., the energy of $X$ is too low and the energy of $X_{S}$ is also too low. It is because $X$ bears $X_{S}$. The disease causal is $X$. 
2) $X$ encounters real disease, and at the same time, $X_{S}$ befalls real disease, i.e., the energy of $X$ is too high and the energy of $X_{S}$ is also too high. It is because $X$ bears $X_{S}$. The disease causal is $X_{S}$.

If intervention reaction coefficients satisfy $\rho_{2}=\rho_{1}^{2}$, it can be shown by Theorem 3.2 that if one wants to treat the abnormal organs $X$ and $X_{S}$, then the following statements are true.

1) For virtual disease of both $X$ and $X_{S}$, the one should intervene organ $X$ directly by increasing its energy. It means, "Virtual disease is to fill his mother" because the disease causal is $X$.

2) For real disease of both $X$ and $X_{S}$, the one should intervene organ $X_{S}$ directly by decreasing its energy. It means, "Real disease is to rush down his son" because the disease causal is $X_{S}$.

The intervention method can be to maintain the balance of the human body because only the energies of two disease organs are changed by using Theorem 3.2, such that there is no side effect for all other organs. And the intervention method can also be to enhance the capability of intervention reaction because the method of using intervention reaction makes the $\rho_{1}$ greater and near to 1 . The state $\rho_{1}=1$ is the best state of the human body system. On the way, it almost has none medical and drug resistance problem since any medicine is possible good for some large $\rho_{1}$.

\subsection{Treatment Principle if Two Organs with the Killing Relation of the Human Body System Encounter Sick}

Suppose that the organs $X$ and $X_{K}$ of a human body system are abnormal (or disease). In the human body system of relations between non-compatible with the constraints, only a situation may occur: $X$ encounters virtual disease, and at the same time, $X_{K}$ befalls real disease, i.e., the energy of $X$ is too low and the energy of $X_{K}$ is too high. It is because it is normal when $X$ kills $X_{K}$ but it is abnormal when $X$ doesn't kill $X_{K}$.

If intervention reaction coefficients satisfy $\rho_{2}=\rho_{1}^{2}$, it can be shown by Theorem 3.4 that if one wants to treat the abnormal organs $X$ and $X_{K}$, the one should intervene organ $X$ directly by increasing its energy, and at the same time, intervene organ $X_{K}$ directly by decreasing its energy. It means that "Strong inhibition of the same time, support the weak".

The intervention method can be to maintain the balance of human body system because only two energies of both disease organs are changed by using Theorem 3.4, such that there is no side effect for all other organs. And the intervention method can also be to enhance the capability of intervention reaction because the method of us- ing intervention reaction makes the $\rho_{1}$ greater and near to 1 such that $X$ can kill $X_{K}$. The state $\rho_{1}=1$ is the best state of the steady multilateral system. On the way, it almost has none medical and drug resistance problem since any medicine is possible good for some large $\rho_{1}$.

\section{Conclusions}

This work shows how to treat the diseases of a human body system and three methods are presented. If only one organ falls ill, mainly the treatment method should be to intervene it indirectly for case: the capability coefficient $\rho_{1} \geq 0.5897545123$ of intervention reaction, according to the treatment principle of "Real disease is to rush down his son but virtual disease is to fill his mother". The intervention method directly can be used in case $\rho_{1}<0.5897545123$, but should be used as little as possible.

If two organs with the loving relation encounter sick, the treatment method should be intervene them directly also according to the treatment principle of "Real disease is to rush down his son but virtual disease is to fill his mother".

If two organs with the killing relation encounter sick, the treatment method should intervene in them directly according to the treatment principle of "Strong inhibition of the same time, support the weak”.

Other properties such as balanced, orderly nature, and so on, will be discussed in the next articles.

\section{Acknowledgements}

This article has been repeatedly invited as reports, such as People's University of China in medical meetings, Shanxi University, Xuchang College, and so on. The work was supported by Specialized Research Fund for the Doctoral Program of Higher Education of Ministry of Education of China (Grant No.44k55050).

\section{References}

[1] Y. S. Zhang, “Multilateral Matrix Theory,” Chinese Statistics Press, 1993. http://www.mlmatrix.com

[2] Y. S. Zhang, S. Q. Pang, Z. M. Jiao and W. Z. Zhao, "Group Partition and Systems of Orthogonal Idempotents,” Linear Algebraand and Its Applications, Vol. 278, 1998, pp. 249-262.

[3] Y. S. Zhang, Y. Q. Lu and S. Q. Pang, “Orthogonal Arrays Obtained by Orthogonal Decomposition of Projection Matrices," Statistica Sinica, Vol. 9, 1999, pp. 595604.

[4] Y. S. Zhang, S. Q. Pang and Y. P. Wang, "Orthogonal Arrays Obtained by Generalized Hadamard Product," 
Discrete Math, Vol. 238, 2001, pp. 151-170. doi:10.1016/S0012-365X(00)00421-0

[5] Y. S. Zhang, L. Duan, Y. Q. Lu and Z. G. Zheng, "Construction of Generalized Hadamard Matrices," Journal of Statistical Planning and Inference, Vol. 104, 2002, pp. 239-258. doi:10.1016/S0378-3758(01)00249-X

[6] Y. S. Zhang and S. S. Mao, “The Origin and Development Philosophy Theory of Statistic," Statistical Research, Vol. 12, 2004, pp. 52-59.

[7] Y. S. Zhang, S. S. Mao, C. Z. Zhan and Z. G. Zheng, "Stable Structure of the Logic Model with Two Causal Effects," Journal of Applied Probability \& Statistics, Vol. 21, No. 4, 2005, pp. 366-374.

[8] N. Q. Feng, Y. H. Qiu, F. Wang, Y. S. Zhang and S. Q. Yin, “A Logic Analysis Model about Complex System's Stability: Enlightenment from Nature,” Lecture Notes in Computer Science, Vol. 3644, 2005, pp. 828-838.

[9] N. Q. Feng, Y. H. Qiu, Y. S. Zhang, F. Wang and Y. He, “A Intelligent Inference Model about Complex System's Stability: Inspiration from Nature,” International Journal of Intelligent Technology, Vol. 1, 2005, pp. 1-6.

[10] N. Q. Feng, Y. H. Qiu, Y. S. Zhang, C. Z. Zhan and Z. G. Zheng, "A Logic Analysis Model of Stability of Complex System Based on Ecology,” Computer Science, Vol. 33, No. 7, 2006, pp. 213-216.

[11] Y. S. Zhang, "Data Analysis and Construction of Orthogonal Arrays,” East China Normal University, 2006.

[12] Y. S. Zhang, "Orthogonal Arrays Obtained by Repeating-Column Difference Matrices,” Discrete Mathematics, Vol. 307, 2007, pp. 246-261. doi:10.1016/j.disc.2006.06.029

[13] C. Y. Pan, X. P. Chen, Y. S. Zhang and S. S. Mao, "Logical Model of Five-Element Theory in Traditional Chinese Medicine," Journal of Chinese Modern Traditional Chinese Medicine, Vol. 4, No. 3, 2008, pp. 193-196.

[14] X. P. Chen, W. J. Zhu, C. Y. Pan and Y. S. Zhang, "Multilateral System,” Journal of Systems Science, Vol. 17, No. 1, 2009, pp. 55-57.

[15] C. Luo and Y. S. Zhang, "Framework Definition and Partition Theorems Dealing with Complex Systems: One of the Series of New Thinking," Journal of Shanghai Institute of Technology (Natural Science), Vol. 10, No. 2, 2010, pp. 109-114.

[16] C. Luo and Y. S. Zhang, "Framework and Orthogonal Arrays: The New Thinking of Dealing with Complex Systems Series Two," Journal of Shanghai Institute of Technology (Natural Science), Vol. 10, No. 3, 2010, pp. 159-163.

[17] X. D. Wang, Y. C. Tang, X. P. Chen and Y. S. Zhang, "Design of Experiment in Global Sensitivity Analysis Based on ANOVA High-Dimensional Model Representation," Communication in Statistics: Simulation and Computation, Vol. 39, 2010, pp. 1183-1195. doi:10.1080/03610918.2010.484122

[18] Research Center for Chinese and Foreign Celebrities and Developing Center of Chinese Culture Resources, "Chinese Philosophy Encyclopedia," Shanghai People Press, 1994.

[19] Y. S. Zhang, “Theory of Multilateral Systems,” 2007. http://www.mlmatrix.com 